\title{
Exact Solitary Wave and Periodic Wave Solutions of a Class of Higher-Order Nonlinear Wave Equations
}

\author{
Lijun Zhang ${ }^{1,2}$ and Chaudry Masood Khalique ${ }^{2}$ \\ ${ }^{1}$ Department of Mathematics, School of Science, Zhejiang Sci-Tech University, Hangzhou, Zhejiang 310018, China \\ ${ }^{2}$ Department of Mathematical Sciences, International Institute for Symmetry Analysis and Mathematical Modelling, \\ North-West University, Mafikeng Campus, Private Bag X 2046, Mmabatho 2735, South Africa
}

Correspondence should be addressed to Lijun Zhang; li-jun0608@163.com

Received 20 February 2015; Revised 5 July 2015; Accepted 26 July 2015

Academic Editor: B. Rush Kumar

Copyright (C) 2015 L. Zhang and C. M. Khalique. This is an open access article distributed under the Creative Commons Attribution License, which permits unrestricted use, distribution, and reproduction in any medium, provided the original work is properly cited.

We study the exact traveling wave solutions of a general fifth-order nonlinear wave equation and a generalized sixth-order KdV equation. We find the solvable lower-order subequations of a general related fourth-order ordinary differential equation involving only even order derivatives and polynomial functions of the dependent variable. It is shown that the exact solitary wave and periodic wave solutions of some high-order nonlinear wave equations can be obtained easily by using this algorithm. As examples, we derive some solitary wave and periodic wave solutions of the Lax equation, the Ito equation, and a general sixth-order KdV equation.

\section{Introduction}

In this paper we study a well-known general fifth-order nonlinear wave equation $[1-5]$

$$
u_{x x x x x}+\alpha u u_{x x x}+\beta u_{x} u_{x x}+\gamma u^{2} u_{x}+u_{t}=0,
$$

where $\alpha, \beta$, and $\gamma$ are real-valued constants, and the general sixth-order KdV equation [6]

$$
\begin{gathered}
u_{x x x x x x}+a u_{x} u_{x x x x}+b u_{x x} u_{x x x}+c u_{x}^{2} u_{x x}+d u_{t t} \\
+e u_{x x x t}+f u_{x} u_{x t}+g u_{t} u_{x x}=0,
\end{gathered}
$$

where $a, b, c, d, e, f$, and $g$ are arbitrary constants.

Equation (1) includes many important nonlinear equations that have been studied in the literature. For example, the Lax equation, the Sawada-Kortera (SK) equation, the Caudrey-Dodd-Gibbon (CDG) equation, the Ito equation, and the Kaup-Kupershmidt (KK) equation are all expressed by (1) in terms of different special values of $\alpha, \beta$, and $\gamma$. Indeed, scaling analysis (by rescaling $u$ to $u / \alpha$ ) shows that only the ratios $\beta / \alpha$ and $\gamma / \alpha^{2}$ matter. The Lax equation $[3,7]$ arises when $\beta / \alpha=2$ and $\gamma / \alpha^{2}=3 / 10$. The KK equation $[2,8-10]$ corresponds to $\beta / \alpha=5 / 2$ and $\gamma / \alpha^{2}=1 / 5$. The SK equation
$[2,9-11]$ and CDG equation [12] are obtained when $\beta / \alpha=1$ and $\gamma / \alpha^{2}=1 / 5$. The Ito equation arises when $\beta / \alpha=2$ and $\gamma / \alpha^{2}=2 / 9$. It has been shown in the literature that the properties of (1) drastically change as $\alpha, \beta$, and $\gamma$ take different values. For instance, the Lax equation and the SK equation are completely integrable and possess $N$-soliton solution. The $\mathrm{KK}$ equation is integrable and has bilinear representation. However, the Ito equation is not completely integrable but has a finite number of conservation laws. For more details, see [1$4,9-11,13]$ and the references in them.

We investigate the traveling wave solutions of the nonlinear wave equation (1) in the form $u(x, t)=y(x-c t)=y(\xi)$, where $c$ is the wave speed and $\xi=x-c t$. Under the traveling wave coordinates, the nonlinear wave equation (1) can be reduced to a nonlinear ordinary differential equation (ODE) of the independent variable $\xi$. Integrating the reduced ODE once with respect to $\xi$ gives

$$
\begin{aligned}
& \frac{d^{4} y}{d \xi^{4}}+\alpha y \frac{d^{2} y}{d \xi^{2}}+\frac{1}{2}(\beta-\alpha)\left(\frac{d y}{d \xi}\right)^{2}+\frac{1}{3} \gamma y^{3}-c y+g \\
& =0,
\end{aligned}
$$

where $g$ is a constant of integration. Clearly, $y(\xi)=y(x-c t)$ is a traveling wave solution of (1) if and only if $y(\xi)$ satisfies 
(3) with the wave speed $c$ and any constant $g$. The equilibrium points and linearized system of (3) were studied in $[7,14,15]$. By using the method of dynamical systems and Cosgrove's results [16], $\mathrm{Li}[7,14,15]$ obtained some exact solitary wave and quasiperiodic wave solutions of the CDG equation.

Generally, we have to study the dynamical behavior of the fourth-order ODE (3) in the 4-dimensional phase space, for which it is usually very difficult to obtain the orbits. However, for the case when the first integrals of this equation can be found, this problem can be reduced to the one in the lower dimensional space which might be easier to handle. It has been a successful idea to find exact solutions of nonlinear PDEs by reducing them into ODEs, especially for some solvable ODEs. A lot of methods in the literatures use this idea, for instance, the tanh-function method and extended tanh-function method [3, 4, 17], simple transformation method [18], the Riccati equation method [19], the Jacobi elliptic function method [20], the expfunction method [21], the homogeneous balance method [22], the $\left(G^{\prime} / G\right)$-expansion method [23], and subequation method [24]. Also a direct and systematical approach to find exact solutions of nonlinear equations was proposed by using rational function transformations and thus was named as the transformed rational function method by $\mathrm{Ma}$ [25]. However, if the dynamics and bifurcation of these ODEs are not investigated, some solutions obtained by different methods, which are presented in different forms, can easily be misunderstood as different solutions [26, 27]. For example, a solution in the form $f\left(\tanh ^{2} \xi\right)$ obtained by the tanh-method can be expressed as $f\left(1-\operatorname{sech}^{2} \xi\right)$ or $f\left(\left(e^{x}-e^{-x}\right)^{2} /\left(e^{x}+e^{-x}\right)^{2}\right)$, which also can be derived by the sech-method and the Expfunction method.

Notice that (3) contains the terms $d^{4} y / d \xi^{4}, d^{2} y / d \xi^{2}$, $(d y / d \xi)^{2}$, and polynomial of $y$. Suppose that $y$ satisfies the equation

$$
\left(\frac{d y}{d \xi}\right)^{2}=P_{m}(y),
$$

where $P_{m}(y)$ is a polynomial function of degree $m$; then

$$
\begin{aligned}
& \frac{d^{2} y}{d \xi^{2}}=\frac{1}{2} P_{m}^{\prime}(y), \\
& \frac{d^{4} y}{d \xi^{4}}=\frac{1}{2} P_{m}^{\prime \prime \prime}(y) P_{m}(y)+\frac{1}{4} P_{m}^{\prime \prime}(y) P_{m}^{\prime}(y),
\end{aligned}
$$

which are both polynomials of $y$. This observation motivates us to try to find some possible integer $m$ and undetermined coefficients of the polynomial $P_{m}$ such that $y$ solves higherorder equation (3) if $y$ is a solution of (4) which is obviously easier to study.

Following the idea we mentioned above, in Section 2, we derive the subequation of a more general fourth-order ODE

$$
\begin{aligned}
& \frac{d^{4} y}{d \xi^{4}}+(A y+B) \frac{d^{2} y}{d \xi^{2}}+C\left(\frac{d y}{d \xi}\right)^{2}+D y^{3}+E y^{2}+F y \\
& +G=0
\end{aligned}
$$

firstly and then investigate the bifurcations and bounded solutions of (7) through the obtained subequations. By the formulas presented in Section 2 and with the help of computer algebra and symbolic computation, we study the bounded traveling wave solutions of the Lax equation and the Ito equation as examples in Section 3. In Section 4, we extend the formulas obtained in Section 2 to study the exact traveling wave solutions of a generalized sixth-order KdV equation.

\section{Subequations and Exact Solutions of the Fourth-Order Equation (7)}

Suppose that $y$ satisfies (4); then the first three terms of the left-hand side of (7) are all polynomials of $y$ and their degrees are $2 m-3, m$ and $m$, respectively. Consequently, we assume $m=3\left(a_{3} \neq 0\right)$ to find the possible polynomial $P_{3}(y)$ such that it solves (7) if $y$ solves (4).

Definition 1. We say that equation $\mathrm{A}$ is a subequation of equation $\mathrm{B}$ if any solutions of equation $\mathrm{A}$ are also solutions of equation $B$.

We now prove that 4 th-order ODE (7) possesses a class of lower-order solvable subequations.

Theorem 2. Suppose that $D \leq(1 / 120)(3 A+2 C)^{2}$. Then $O D E$ (7) has the following subequation:

$$
\left(\frac{d y}{d \xi}\right)^{2}=P_{3}(y)=a_{3} y^{3}+a_{2} y^{2}+a_{1} y+a_{0}
$$

where

$$
\begin{aligned}
& a_{3}=-\frac{1}{30}\left[(3 A+2 C) \pm \sqrt{(3 A+2 C)^{2}-120 D}\right] \\
& a_{2}=-\frac{3 B a_{3}+2 E}{2 C+15 a_{3}+2 A} \\
& a_{1}=-\frac{2\left(B a_{2}+a_{2}^{2}+F\right)}{9 a_{3}+2 C+A} \\
& a_{0}=-\frac{B a_{1}+a_{1} a_{2}+2 G}{2\left(C+3 a_{3}\right)} .
\end{aligned}
$$

That is, the function $y=y(\xi)$ solves the fourth-order differential equation (7) if it solves (8).

Proof. By differentiating (8) with respect to $\xi$ once and three times, we obtain $d^{2} y / d \xi^{2}=(1 / 2) P_{3}^{\prime}(y)$ and $d^{4} y / d \xi^{4}=$ $(1 / 2) P_{3}^{\prime \prime \prime}(y) P_{3}(y)+(1 / 4) P_{3}^{\prime \prime}(y) P_{3}^{\prime}(y)$ which are (5) and (6) with $m=3$, respectively. Inserting (8), (5), and (6) with $m=3$ 
into (7) and comparing the coefficients of like powers of $y$, we have

$$
\begin{array}{r}
y^{3}: D+\frac{3}{2} A a_{3}+\frac{15}{2} a_{3}^{2}+C a_{3}=0, \\
y^{2}: C a_{2}+\frac{3}{2} B a_{3}+\frac{15}{2} a_{2} a_{3}+A a_{2}+E=0, \\
y^{1}: F+\frac{9}{2} a_{1} a_{3}+a_{2}^{2}+B a_{2}+C a_{1}+\frac{1}{2} A a_{1}=0, \\
y^{0}: \frac{1}{2} a_{1} a_{2}+C a_{0}+3 a_{0} a_{3}+\frac{1}{2} B a_{1}+G=0 .
\end{array}
$$

Solving system (10) gives us (9).

Remark 3. Note that all the denominators in (9) are supposed to be nonzero. If some of them are zero, we have to go back to the algebraic equations (10) to find the possible solutions.

According to the conclusion of Theorem 2, we know that the fourth-order ODE (7) can be reduced into the first-order nonlinear ODE (8) provided that $D \leq(3 A+2 C)^{2} / 120$, which is really an inspiring result because the bifurcation and the exact solutions of the first-order nonlinear ODE (8) have been presented in [8]. We recall the theorem below to derive the exact solutions of (7) and thus obtain the exact traveling wave solutions of the fifth-order nonlinear wave equation (1) and the sixth-order KdV equation (2).

Theorem 4. Let $h_{ \pm}=\left(2 \Delta\left(-a_{2} \pm \sqrt{\Delta}\right)+3 a_{1} a_{2} a_{3}\right) / 54 a_{3}^{2}$ and $y_{e}^{ \pm}=\left(-a_{2} \pm \sqrt{\Delta}\right) / 3 a_{3}$, where $\Delta=a_{2}^{2}-3 a_{1} a_{3}>0$; then the following conclusions hold.

(1) For $a_{0}=2 h_{+}$, (8) has a bounded solution approaching $y_{e}^{+}$as $\xi$ goes to infinity given by

$$
y=\frac{-a_{2}+\sqrt{\Delta}}{3 a_{3}}-\frac{\sqrt{\Delta}}{a_{3}} \operatorname{sech}^{2}\left[\frac{1}{2} \Delta^{1 / 4}\left(\xi-\xi_{0}\right)\right],
$$

a constant solution

$$
y=\frac{-a_{2}+\sqrt{\Delta}}{3 a_{3}},
$$

and an unbounded solution

$$
y=\frac{-a_{2}+\sqrt{\Delta}}{3 a_{3}}+\frac{\sqrt{\Delta}}{a_{3}} \operatorname{csch}^{2}\left[\frac{1}{2} \Delta^{1 / 4}\left(\xi-\xi_{0}\right)\right],
$$

where $\xi_{0}$ is an arbitrary constant.

(2) Suppose that $a_{0} \in\left(2 h_{-}, 2 h_{+}\right)$.

Case (a). If $a_{3}>0$, then, for any $y_{3} \in\left(-\left(a_{2}+2 \sqrt{\Delta}\right) / 3 a_{3},-\left(a_{2}+\right.\right.$ $\left.\sqrt{\Delta}) / 3 a_{3}\right)$,

$$
\begin{aligned}
y= & y_{3} \\
& -\frac{1}{2}\left(3 y_{3}+\frac{a_{2}}{a_{3}}+\sqrt{\Delta_{+}}\right) \operatorname{sn}^{2}\left(\Omega_{+}\left(\xi-\xi_{0}\right), k_{+}\right),
\end{aligned}
$$

is a family of smooth periodic solutions of (8). Here $k_{+}=$ $2 \sqrt{3 y_{3}^{2}+2\left(a_{2} / a_{3}\right) y_{3}+a_{1} / a_{3}} /\left(-3 y_{3}-a_{2} / a_{3}+\sqrt{\Delta_{+}}\right), \Omega_{+}=$ $(\sqrt{2} / 4) \sqrt{-3 a_{3} y_{3}-a_{2}+a_{3} \sqrt{\Delta_{+}}}, \Delta_{+}=-3 y_{3}^{2}-2\left(a_{2} / a_{3}\right) y_{3}+$ $\left(a_{2} / a_{3}\right)^{2}-4\left(a_{1} / a_{3}\right)$, and sn represents the Jacobian elliptic sineamplitude function.

Case (b). If $a_{3}<0$, then, for any $y_{1} \in\left(-\left(a_{2}+\sqrt{\Delta}\right) / 3 a_{3},-\left(a_{2}+\right.\right.$ $\left.2 \sqrt{\Delta}) / 3 a_{3}\right)$,

$$
\begin{aligned}
y= & y_{1} \\
& -\frac{1}{2}\left(3 y_{1}+\frac{a_{2}}{a_{3}}-\sqrt{\Delta_{-}}\right) \operatorname{sn}^{2}\left(\Omega_{-}\left(\xi-\xi_{0}\right), k_{-}\right),
\end{aligned}
$$

is a family of smooth periodic solutions of (8). Here $k_{-}=$ $2 \sqrt{3 y_{1}^{2}+2\left(a_{2} / a_{3}\right) y_{1}+a_{1} / a_{3}} /\left(3 y_{1}+a_{2} / a_{3}+\sqrt{\Delta_{-}}\right)$and $\Omega_{-}=$ $(\sqrt{2} / 4) \sqrt{-3 a_{3} y_{1}-a_{2}-a_{3} \sqrt{\Delta_{-}}}$and $\Delta_{-}=-3 y_{1}^{2}-2\left(a_{2} / a_{3}\right) y_{1}+$ $\left(a_{2} / a_{3}\right)^{2}-4\left(a_{1} / a_{3}\right)$.

(3) For $a_{0} \in\left(-\infty, 2 h_{-}\right] \cup\left(2 h_{+},+\infty\right)$, (8) has no nontrivial bounded solutions. When $a_{0}=2 h_{-}$, an unbounded solution is given by

$$
y=-\frac{a_{2}+\sqrt{\Delta}}{3 a_{3}}+\frac{\sqrt{\Delta}}{a_{3}} \sec ^{2}\left[\frac{1}{2} \Delta^{1 / 4}\left(\xi-\xi_{0}\right)\right],
$$

and a constant solution is given by

$$
y=-\frac{a_{2}+\sqrt{\Delta}}{3 a_{3}} .
$$

Remark 5. From Theorem 4, we conclude that (11)-(17) are solutions of the second-order ODE $d^{2} y / d \xi^{2}=(1 / 2) P_{3}^{\prime}(y)=$ $(1 / 2)\left(3 a_{3} y^{2}+2 a_{2} y+a_{1}\right)$.

Inserting $A=\alpha, B=0, C=(\beta-\alpha) / 2, D=\gamma / 3, E=$ $0, F=-c$, and $G=g$ into (7) gives (3). Consequently, in terms of Theorem 2, we get the subequation of (3).

Theorem 6. Suppose that $\gamma \leq(2 \alpha+\beta)^{2} / 40$ and $\gamma \notin\left\{-3 \alpha^{2} /\right.$ $\left.10-\alpha \beta / 2-\beta^{2} / 5,-9 \alpha^{2} / 8+3 \alpha \beta / 2-3 \beta^{2} / 8, \alpha \beta / 3-\beta^{2} / 9\right\}$. Then ODE (3) admits the subequation (8) with

$$
\begin{aligned}
& a_{3}=-\frac{1}{30}\left[(2 \alpha+\beta) \pm \sqrt{(2 \alpha+\beta)^{2}-40 \gamma}\right] \\
& a_{2}=0 \\
& a_{1}=\frac{2 c}{9 a_{3}+\beta}, \\
& a_{0}=\frac{2 g}{\alpha-\beta-6 a_{3}} .
\end{aligned}
$$

From the proof of Theorem 2, one can easily find that the undetermined coefficients of the subequation (8) are determined by the equations corresponding to (10). Thus we may find more solutions for the case when the denominators of (18) are 0 . If $\gamma \leq(2 \alpha+\beta)^{2} / 40$ and $\gamma \notin\left\{-3 \alpha^{2} / 10-\alpha \beta / 2-\right.$ $\left.\beta^{2} / 5,-9 \alpha^{2} / 8+3 \alpha \beta / 2-3 \beta^{2} / 8, \alpha \beta / 3-\beta^{2} / 9\right\}$, then $a_{3} \notin\{-(\alpha+$ $\beta) / 15,(\alpha-\beta) / 6,-\beta / 9\}$. Then the determining equations of $a_{0}, a_{1}$, and $a_{2}$ will have unique solutions for the determined 
$a_{3}$. However, for the case when $\gamma=-3 \alpha^{2} / 10-\alpha \beta / 2-\beta^{2} / 5$ and thus $a_{3}=-(\alpha+\beta) / 15, a_{2}$ can be arbitrary constant; for the case when $\gamma=-9 \alpha^{2} / 8+3 \alpha \beta / 2-3 \beta^{2} / 8$ and thus $a_{3}=(\alpha-\beta) / 6, a_{0}$ can be arbitrary constant if $g$ is chosen to be zero; for $\gamma=\alpha \beta / 3-\beta^{2} / 9$ and thus $a_{3}=-\beta / 9, a_{0}$ can be arbitrary constant if $c$ is chosen to be zero.

\section{Exact Traveling Wave Solutions of a Class of 5th-Order Nonlinear Wave Equations}

In Section 2, we derived some subequations of the fourthorder ODE (3) which determines the traveling wave solutions of the fifth-order nonlinear wave equation (1). Therefore, one can derive the exact traveling wave solutions of (1) through Theorems 4 and 6 . Note that $a_{0}$ is determined by an arbitrary integration constant $g$, so $a_{0}$ is also an arbitrary constant. Clearly,

$$
\begin{aligned}
\Delta_{ \pm} & =a_{2}^{2}-3 a_{1} a_{3} \\
& =\frac{2 c\left(-(2 \alpha+\beta) \pm \sqrt{(2 \alpha+\beta)^{2}-40 \gamma}\right)}{7 \alpha-4 \beta \mp \sqrt{(2 \alpha+\beta)^{2}-40 \gamma}} .
\end{aligned}
$$

According to Theorem 4, if $2 c(-(2 \alpha+\beta) \pm$ $\sqrt{\left.(2 \alpha+\beta)^{2}-40 \gamma\right)} /\left(7 \alpha-4 \beta \mp \sqrt{\left.(2 \alpha+\beta)^{2}-40 \gamma\right)}>0\right.$, the bounded solutions of (4) can be obtained from (11)-(17).

We now study the Lax equation and the Ito equation as examples of the application of the approach we proposed in this paper.

3.1. Exact Traveling Wave Solutions of the Lax Equation. The Lax equation which has been studied in $[3,7]$ is given by

$$
u_{x x x x x}+10 u u_{x x x}+20 u_{x} u_{x x}+30 u^{2} u_{x}+u_{t}=0 .
$$

This is (1) with $\alpha=10, \beta=20$, and $\gamma=30$.

Theorem 7. The Lax equation (20) has the following four families of bounded traveling wave solutions.

(1) The Lax equation (20) has a peak-form solitary wave solution (see Figure 1)

$$
\begin{aligned}
u(x, t)= & \frac{-\sqrt{14 c}}{14} \\
& +\frac{3 \sqrt{14 c}}{14} \operatorname{sech}^{2}\left[\frac{1}{2}\left(\frac{2}{7} c\right)^{1 / 4}\left(x-c t-\xi_{0}\right)\right],
\end{aligned}
$$

where the wave speed $c>0$.

(2) For any arbitrary constant $a_{2}$, the Lax equation (20) has a family of peak-form solitary wave solutions

$$
\begin{gathered}
u(x, t)=\frac{1}{6}\left(a_{2}-\sqrt{6 c-5 a_{2}^{2}}\right)+\frac{1}{2} \sqrt{6 c-5 a_{2}^{2}} \\
\cdot \operatorname{sech}^{2}\left[\frac{1}{2}\left(6 c-5 a_{2}^{2}\right)^{1 / 4}\left(x-c t-\xi_{0}\right)\right],
\end{gathered}
$$

where $c>(5 / 6) a_{2}^{2}$.

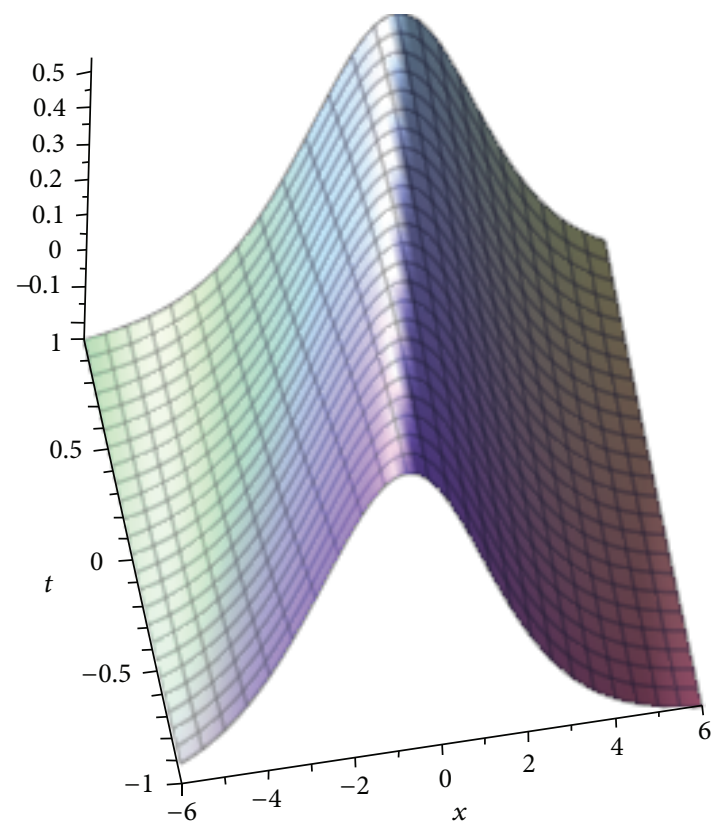

FIGURE 1: Three-dimensional portrait of the solitary wave solution of the Lax equation (20) with $c=1$.

(3) For any arbitrary $c>0$ and $u_{1} \in((1 / 14) \sqrt{14 c}$, $(1 / 7) \sqrt{14 c})$,

$$
\begin{aligned}
u(x, t) & \\
= & u_{1} \\
& +\frac{1}{2}\left(\sqrt{\Delta_{1}}-3 u_{1}\right) \operatorname{sn}^{2}\left(\Omega_{1}\left(x-c t-\xi_{0}\right), k_{1}\right),
\end{aligned}
$$

is a family of smooth periodic traveling wave solutions of the Lax equation (20). Here $\Omega_{1}=(1 / 6) \sqrt{9 u_{1}+3 \sqrt{\Delta_{1}}}, k_{1}=$ $\sqrt{12 u_{1}^{2}-(6 / 7) c} /\left(3 u_{1}+\sqrt{\Delta_{1}}\right)$, and $\Delta_{1}=(6 / 7) c-3 u_{1}^{2}$.

(4) For any arbitrary $a_{2}, c>(5 / 6) a_{2}^{2}$ and $u_{1} \in\left((1 / 6)\left(a_{2}+\right.\right.$ $\left.\left.\sqrt{6 c-5 a_{2}^{2}}\right),(1 / 6)\left(a_{2}+2 \sqrt{6 c-5 a_{2}^{2}}\right)\right)$,

$$
\begin{array}{r}
u(x, t)=u_{1}+\frac{1}{2}\left(3 u_{1}+\frac{1}{2} a_{2}+\sqrt{\Delta_{2}}\right) \\
\cdot \operatorname{sn}^{2}\left(\Omega_{2}\left(x-c t-\xi_{0}\right), k_{2}\right)
\end{array}
$$

is a family of smooth periodic traveling wave solutions of the Lax equation (20) (see Figure 2). Here $\Delta_{2}=2 c-3 u_{1}^{2}+a_{2} u_{1}$ $(7 / 4) a_{2}^{2}, \Omega_{2}=(1 / 4) \sqrt{12 u_{1}-2 a_{2}+4 \sqrt{\Delta_{2}}}$, and $k_{2}=$ $2 \sqrt{12 u_{1}^{2}-4 a_{2} u_{1}+2 a_{2}^{2}-2 c} /\left(6 u_{1}-a_{2}+2 \sqrt{\Delta_{2}}\right)$.

Proof. Inserting $\alpha=10, \beta=20$, and $\gamma=30$ into the first equation of (18) gives $a_{3}=2 / 3$ and $a_{3}=-2$. Note that $(1 / 15)(\alpha+\beta)=2$. Using these values with $a_{3}=-2 / 3,(18)$ and (19) give $a_{2}=0, a_{1}=(1 / 7) c, a_{0}=-(1 / 3) g$, and $\Delta_{+}=(2 / 7) c$ and so from (11), we obtain (21) which is a peak-form solitary wave solution of the Lax equation. 


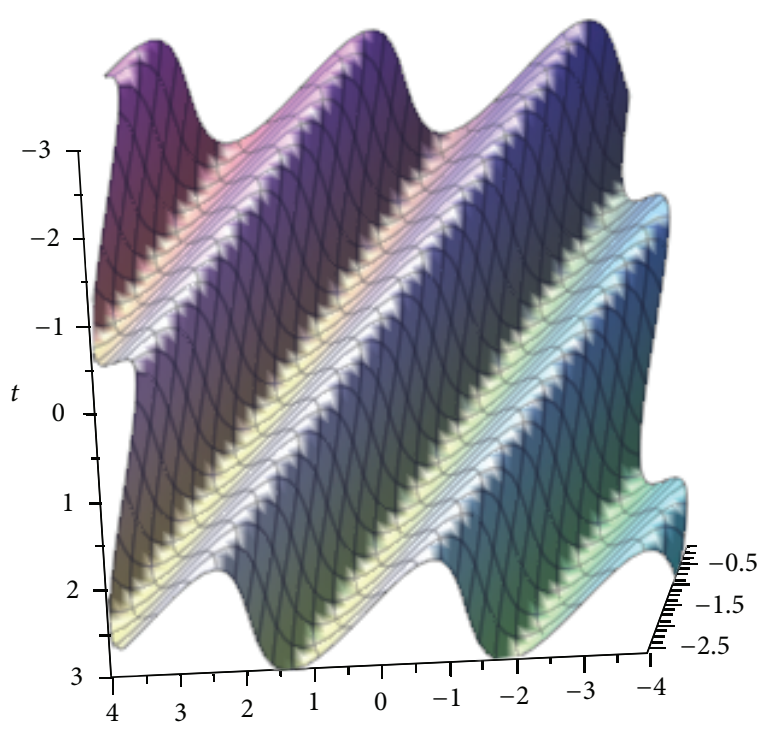

(a)

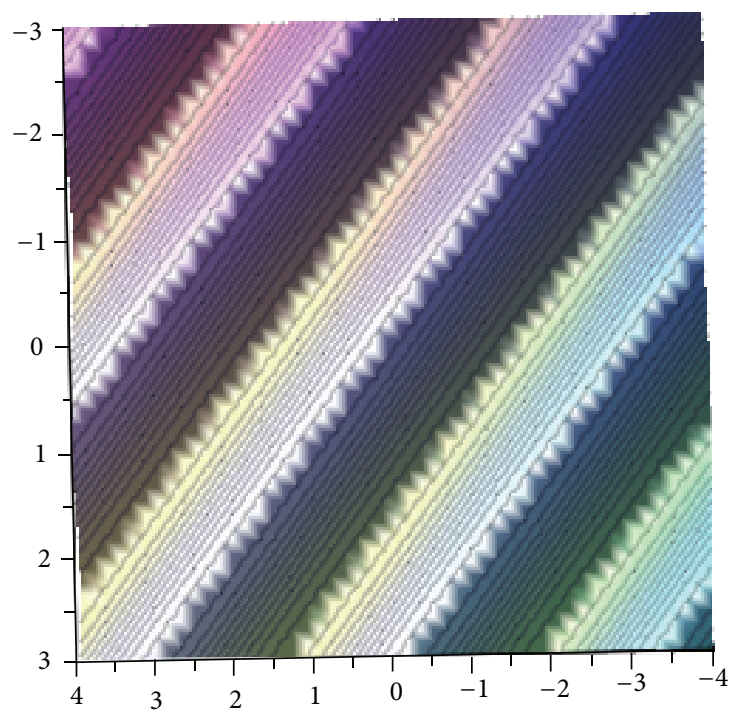

(b)

FIGURE 2: Portrait of the periodic wave solution of the Lax equation (20) with $c=1$. (a) Three-dimensional portrait; (b) overhead view with contour plot.

Further, since $g$ is an arbitrary constant, we suppose that $(2 / 3) u^{3}-(1 / 7) c u+(1 / 3) g=(2 / 3)\left(u_{1}-u\right)\left(u_{2}-u\right)\left(u-u_{3}\right)$ for certain value of $g$, where $u_{1}>u_{2}>u_{3}$. Then by the relationship between coefficients and roots of a polynomial, we have

$$
\begin{aligned}
u_{1}+u_{2}+u_{3} & =0, \\
u_{1} u_{2}+u_{1} u_{3}+u_{2} u_{3} & =-\frac{3}{14} c .
\end{aligned}
$$

Obviously, $(1 / 14) \sqrt{14 c}<u_{1}<(1 / 7) \sqrt{14 c}$. By letting $\Delta_{1}=(6 / 7) c-3 u_{1}^{2}$, from (25), we obtain

$$
\begin{aligned}
& u_{2}=-\frac{1}{2}\left(u_{1}-\sqrt{\Delta_{1}}\right), \\
& u_{3}=-\frac{1}{2}\left(u_{1}+\sqrt{\Delta_{1}}\right) .
\end{aligned}
$$

Inserting (26) into (15) gives (23).

For the second value of $a_{3}=-2$, we note that the denominator and numerator of $a_{2}$ in (9) are both zero for $\alpha=10, \beta=20$, and $\gamma=30$. Thus we have to go back to the algebraic equations (10). Clearly, $A=10, B=0, C=5$, and $D=10$ when $\alpha=10, \beta=20$, and $\gamma=30$. Substituting $a_{3}=-2$ into (10) gives

$$
\begin{aligned}
a_{2}^{2}+a_{1}-c & =0, \\
\frac{1}{2} a_{1} a_{2}-a_{0}+g & =0 .
\end{aligned}
$$

Solving (27) for $a_{2}, a_{1}$, and $a_{0}$ gives $a_{1}=c-a_{2}^{2}$ and $a_{0}=$ $g+(1 / 2) a_{2} c-(1 / 2) a_{2}^{3}$ and $a_{2}$ can be an arbitrary constant. Inserting these results into (11) and (14) gives (22) and (24), respectively.
Remark 8. We note that we recover the solutions of the Lax equation obtained in [3] as special cases of our solutions. The solitary wave solutions (22) are consistent with the solutions (138) in [3], but the solution (21) and other two families of periodic wave solutions are new.

3.2. Exact Traveling Wave Solutions of the Ito Equation. The Ito equation [13] is given by

$$
u_{x x x x x}+3 u u_{x x x}+6 u_{x} u_{x x}+2 u^{2} u_{x}+u_{t}=0
$$

and can be retrieved from (1) by letting $\alpha=3, \beta=6$, and $\gamma=2$.

Theorem 9. The Ito equation (28) has the following four families of bounded traveling wave solutions.

(1) It has a peak-form solitary wave solution

$$
\begin{aligned}
u(x, t)= & -\frac{5}{12} \sqrt{6 c} \\
& +\frac{5}{4} \sqrt{6 c} \operatorname{sech}^{2}\left[\frac{1}{2}\left(\frac{c}{6}\right)^{1 / 4}\left(x-c t-\xi_{0}\right)\right],
\end{aligned}
$$

where the wave speed $c>0$.

(2) For any arbitrary constant $u_{0}$, the Ito equation (28) has a family of peak-form solitary wave solutions

$$
u(x, t)=-2 u_{0}^{2}+6 u_{0}^{2} \operatorname{sech}^{2}\left[u_{0}\left(x-\xi_{0}\right)\right] .
$$

The wave speed of this family of waves is zero; that is, these are standing waves. 
(3) For any arbitrary $c>0$ and $u_{1} \in((5 / 12) \sqrt{6 c}$, $(5 / 6) \sqrt{6 c})$,

$$
\begin{aligned}
& u(x, t) \\
& \quad=u_{1}+\frac{1}{2}\left(\sqrt{\Delta_{3}}-3 u_{1}\right) \operatorname{sn}^{2}\left(\Omega_{3}\left(x-c t-\xi_{0}\right), k_{3}\right),
\end{aligned}
$$

is a family of smooth periodic traveling wave solutions of the Ito equation. Here $\Omega_{3}=(1 / 60) \sqrt{60\left(3 u_{1}+\sqrt{\Delta_{3}}\right)}, k_{3}=$ $\sqrt{48 u_{1}^{2}-50 c} /\left(6 u_{1}+2 \sqrt{\Delta_{3}}\right)$, and $\Delta_{3}=(25 / 2) c-3 u_{1}^{2}$.

(4) For any arbitrary $a_{1}>0$ and $u_{1} \in\left((1 / 2) \sqrt{2 a_{1}}, \sqrt{2 a_{1}}\right)$,

$$
u(x, t)=u_{1}+\frac{1}{2}\left(\sqrt{\Delta_{4}}-3 u_{1}\right) \operatorname{sn}^{2}\left(\Omega_{4}\left(x-\xi_{0}\right), k_{4}\right)
$$

is a family of smooth periodic traveling wave solutions of the (28). Here $\Delta_{4}=6 a_{1}-3 u_{1}^{2}, \Omega_{4}=(1 / 6) \sqrt{9 u_{1}+3 \sqrt{\Delta_{4}}}$, and $k_{4}=\sqrt{12 u_{1}^{2}-6 a_{1}} /\left(3 u_{1}+\sqrt{\Delta_{4}}\right)$.

Proof. Inserting $\alpha=3, \beta=6$, and $\gamma=2$ into the first equation of (19) gives $a_{3}=-2 / 15$ or $a_{3}=-2 / 3$. Using the values of $\alpha, \beta$, and $\gamma$ with $a_{3}=-2 / 15,(19)$ gives $a_{2}=0$ and $a_{1}=(5 / 12) c$. Thus $\Delta_{+}=(1 / 6) c$. Inserting these results into (11) gives (29) which is a peak-form solitary wave solution of the Ito equation.

Further, we suppose that $-(2 / 15) u^{3}+(1 / 7) c u-(1 / 3) g=$ $-(2 / 15)\left(u_{1}-u\right)\left(u_{2}-u\right)\left(u-u_{3}\right)$ for certain value of $g$, where $u_{1}>u_{2}>u_{3}$. Using the relationship between coefficients and roots of a polynomial, we have

$$
\begin{aligned}
u_{1}+u_{2}+u_{3} & =0 \\
u_{1} u_{2}+u_{1} u_{3}+u_{2} u_{3} & =-\frac{25}{8} c .
\end{aligned}
$$

Clearly, $(5 / 12) \sqrt{6 c}<u_{1}<(5 / 6) \sqrt{6 c}$. Letting $\Delta_{3}=50 c-$ $12 u_{1}^{2}$, from (33), we obtain

$$
\begin{aligned}
& u_{2}=-\frac{1}{4}\left(2 u_{1}-\sqrt{\Delta_{3}}\right), \\
& u_{3}=-\frac{1}{4}\left(2 u_{1}+\sqrt{\Delta_{3}}\right) .
\end{aligned}
$$

Inserting (34) into (15) gives (31).

Now, for $a_{3}=-2 / 3$, the denominator of $a_{1}$ in (9) is zero when $\alpha=3, \beta=6$, and $\gamma=2$, so we have to go back to the algebraic equations (10). Clearly, $A=3, B=0, C=3 / 2, D=$ 13 , and $E=0$ when $\alpha=3, \beta=6$, and $\gamma=2$. From the second equation of (10), we obtain $a_{2}=0$. Clearly, when $a_{3}=-2 / 3$ and $a_{2}=0$, any arbitrary $a_{1}$ solves the third equation of (10) if $F=0$; that is, $c=0$. Then from the last equation of (10), we obtain $a_{0}=2 g$. Using these values into (11) and (15) we obtain (30) and (32), respectively.

Remark 10. The solitary wave solutions (29) and (30) are consistent with the solutions (89) and (91) in [3], respectively. However, the periodic solutions (31) and (32) are new.

\section{Application to the General Sixth-Order KdV Equation (2)}

We now generalize this approach to study a new sixth-order nonlinear wave equation (2) which was derived by KarasuKalkanli et al. [6] in 2008. Letting $u(x, t)=u(x-v t)=u(\xi)$, setting $y=d u / d \xi$, and integrating it once with respect to $\xi$, we have

$$
\begin{gathered}
\frac{d^{4} y}{d \xi^{4}}+(a y-e v) \frac{d^{2} y}{d \xi^{2}}+\frac{1}{2}(b-a)\left(\frac{d y}{d \xi}\right)^{2}+\frac{1}{3} c y^{3} \\
-\frac{1}{2} v(f+g) y^{2}+d v^{2} y+\beta=0,
\end{gathered}
$$

where $\beta$ is an integration constant.

Obviously, the traveling wave solutions of the sixth-order $\mathrm{KdV}$ equation (2) are given by $u(\xi)=\int y(\xi) d \xi$, where $y(\xi)$ solves ODE (35) which is similar to (7). By letting $A=a$, $B=-e v, C=(1 / 2)(b-a), D=(1 / 3) c, E=-(1 / 2) v(f+g)$, $F=d v^{2}$, and $G=\beta$ in (9), we have the following lemma.

Lemma 11. Suppose $c \leq(1 / 40)(b+2 a)^{2}$. If $y=y(\xi)$ solves (35), then it solves (8), where

$$
\begin{aligned}
& a_{3}=-\frac{1}{30}\left[b+2 a \pm \sqrt{(b+2 a)^{2}-40 c}\right], \\
& a_{2}=\frac{v(f+g)+3 e v a_{3}}{15 a_{3}+b+a}, \\
& a_{1}=\frac{2\left(e v a_{2}-d v^{2}-a_{2}^{2}\right)}{9 a_{3}+b}, \\
& a_{0}=\frac{e v a_{1}-a_{1} a_{2}-2 \beta}{6 a_{3}+b-a} .
\end{aligned}
$$

Remark 12. If any denominators of $a_{2}, a_{1}$, or $a_{0}$ are zero, we need to go back to the equations

$$
\begin{aligned}
y^{3} & : 15 a_{3}^{2}+3 a a_{3}+(b-a) a_{3}+\frac{2}{3} c=0, \\
y^{2}: & 15 a_{2} a_{3}+4 a a_{2}-3 a_{3} e v+(b-a) a_{2}-v(f+g) \\
& =0, \\
y^{1}: & \frac{9}{2} a_{1} a_{3}+a_{2}^{2}-2 a_{2} e v+a a_{1}+\frac{1}{2}(b-a) a_{1}+d v^{2} \\
\quad= & 0, \\
y^{0}: & a_{1} a_{2}+6 a_{0} a_{3}-2 a_{1} e v+(b-a) a_{0}+2 \beta=0
\end{aligned}
$$

to find possible solutions.

Note that here $a_{0}$ can be arbitrary number because $\beta$ is an arbitrary integration constant. From Lemma 11 and Theorem 4, it is easy to see that the traveling wave solutions of the six-order KdV equations (2) can be obtained by detecting the relationship between the coefficients $a_{i}, i=0,1,2,3$, which are determined by (36). The two families of exact 
solutions exist if $a_{1}, a_{2}$, and $a_{3}$ defined by (36) satisfy $a_{2}^{2}-$ $3 a_{1} a_{3}>0$, because $a_{0}$ is an integration constant. With the help of Maple, we can obtain the traveling waves of the sixorder KdV equation (2) automatically.

Equation (2) with $a=20, b=40, c=120, d=0, e=1$, $f=8$, and $g=4$, namely,

$$
\begin{gathered}
u_{x x x x x x}+20 u_{x} u_{x x x x}+40 u_{x x} u_{x x x}+120 u_{x}^{2} u_{x x} \\
+u_{x x x t}+8 u_{x}^{2} u_{x t}+4 u_{t}^{2} u_{x x}=0
\end{gathered}
$$

was studied in [28]. According to (36) and Theorem 4, we obtain $a_{3}=-4 / 3, a_{2}=(1 / 5) v, a_{1}=(2 / 175) v^{2}, a_{0}=$ $(2 / 2625) v^{3}-(1 / 6) \beta$, or $a_{3}=-4, a_{1}=(1 / 2)\left(v a_{2}-a_{2}^{2}\right)$, and $a_{0}=(1 / 8)\left(2 v a_{2}^{2}-v^{2} a_{2}-a_{2}^{3}+4 \beta\right)$, and $a_{2}$ is an arbitrary constant.

Note that $\beta$ is an arbitrary integration constant. Consequently, from Theorem 4, we obtain the exact solutions of (38) as $u(x, t)=\int y(\xi) d \xi$, where $\xi=x-v t$ and $y(\xi)$ is defined as follows.

(1) Consider

$$
\begin{aligned}
y= & \frac{1}{140}(7 v-\sqrt{105}|v|) \\
& +\frac{3}{140} \sqrt{105}|v| \operatorname{sech}^{2}\left(\frac{1}{4}\left(\frac{3}{35} v^{2}\right)^{1 / 4}\left(\xi-\xi_{0}\right)\right) .
\end{aligned}
$$

(2) For arbitrary $a_{2}$,

$$
\begin{gathered}
y=\frac{1}{12}\left(a_{2}-\sqrt{-5 a_{2}^{2}+6 a_{2} v}\right)+\frac{1}{4} \sqrt{6 a_{2} v-5 a_{2}^{2}} \\
\cdot \operatorname{sech}^{2}\left(\frac{1}{2}\left(-5 a_{2}^{2}+6 a_{2} v\right)^{1 / 4}\left(\xi-\xi_{0}\right)\right) .
\end{gathered}
$$

(3) For any arbitrary $v \neq 0$ and $(1 / 140)(7 v+\sqrt{105}|v|)<$ $y_{1}<(1 / 140)(7 v+2 \sqrt{105}|v|)$,

$$
\begin{aligned}
y= & y_{1} \\
& +\left(\frac{3}{2} y_{1}+\frac{3}{40} v+\frac{1}{2} \sqrt{\Delta_{5}}\right) \operatorname{sn}^{2}\left(\Omega_{5}\left(\xi-\xi_{0}\right), k_{5}\right),
\end{aligned}
$$

where $\Omega_{5}=(1 / 60) \sqrt{1800 y_{1}-90 v+600 \sqrt{\Delta_{5}}}, k_{5}=$ $\sqrt{-42 v^{2}-1470 v y_{1}+14700 y_{1}^{2}} / 35\left(3 y_{1}-(3 / 20) v+\sqrt{\Delta_{5}}\right)$, and $\Delta_{5}=(3 / 10) v y_{1}+(159 / 2800) v^{2}-3 y_{1}^{2}$.

(4) For any arbitrary $a_{2}, v \neq 0$, and $(1 / 12)\left(a_{2}+\right.$ $\left.\sqrt{-5 a_{2}^{2}+6 v a_{2}}\right)<y_{1}<(1 / 12)\left(a_{2}+2 \sqrt{-5 a_{2}^{2}+6 v a_{2}}\right)$,

$$
\begin{aligned}
y= & y_{1} \\
& +\left(\frac{3}{2} y_{1}+\frac{1}{8} a_{2}+\frac{1}{2} \sqrt{\Delta_{6}}\right) \operatorname{sn}^{2}\left(\Omega_{6}\left(\xi-\xi_{0}\right), k_{6}\right),
\end{aligned}
$$

where $\Omega_{6}=(1 / 4) \sqrt{24 y_{1}-2 a_{2}+8 \sqrt{\Delta_{6}}}, k_{6}=$ $\sqrt{48 y_{1}^{2}-8 a_{2} y_{1}-2 a_{2} v+2 a_{2}^{2}} /\left(6 y_{1}-(1 / 2) a_{2}+2 \sqrt{\Delta_{6}}\right)$, and $\Delta_{6}=(1 / 2) a_{2} y_{1}-7 a_{2}^{2}+(1 / 2) v a_{2}-3 y_{1}^{2}$.

Remark 13. Obviously, this algorithm also can be used to study the sixth-order KdV equation (2) with other coefficients values.

\section{Conclusion and Discussion}

The exact traveling wave solutions of a general fifth-order nonlinear wave equation and a generalized sixth-order $\mathrm{KdV}$ equation were studied in this paper. A systematic algorithm was proposed to study the exact solutions of an associated fourth-order ODE possessing even order derivatives. By using this algorithm, with the help of symbolic computation, the exact solitary wave and periodic wave solutions of a very general class of higher-order nonlinear wave equations can be obtained systematically. Here we obtained two families of solitary wave and periodic wave solutions of the Lax equation, the Ito equation, and a generalized sixth-order KdV equation. From the results of this paper, it is easy to see that this method can be used to find the traveling wave solutions of higherorder wave equations which can be reduced to the ODEs in the form $F\left(y^{(2 k)}, y^{(2 k-2)}, \ldots, y^{(2)}, y^{\prime 2}, y\right)=0$, where $F$ is a polynomial function.

It has been shown that the equations with nonlinear dispersion, usually related to singular dynamical systems, possess nonsmooth singular wave solutions, such as compacton and peakon, by using the dynamical system method and other methods [14, 29-32]. However, as far as we know, the dynamical system method has been well used to investigate the nonlinear wave equations which can be reduced to planar dynamical systems. Can we find the subequations of the higher-order wave equations with nonlinear dispersion in the form $(d y / d \xi)^{2}=R(y)$ ? Here $R(y)$ is a rational function. How the theorems of planar singular dynamical systems $[14,33,34]$ can be applied to find the singular wave solutions to higherorder nonlinear wave equations will be considered in our future work.

\section{Conflict of Interests}

The authors declare that there is no conflict of interests regarding the publication of this paper.

\section{Acknowledgments}

Lijun Zhang thanks the North-West University for the postdoctoral fellowship and DST-NRF Centre of Excellence in Mathematical and Statistical Sciences (CoE-MaSS). This work is partially supported by the Nature Science Foundation of China (nos. 11101371 and 11422214) and the Nature Science Foundation of Zhejiang (LY15A010021). The authors are also grateful to the referees for their valuable comments, which have led to several improvements.

\section{References}

[1] W. Hereman and A. Nuseir, "Symbolic methods to construct exact solutions of nonlinear partial differential equations," Mathematics and Computers in Simulation, vol. 43, no. 1, pp. 1327, 1997.

[2] M. Inc, "On numerical soliton solution of the KaupKupershmidt equation and convergence analysis of the decomposition method," Applied Mathematics and Computation, vol. 172, no. 1, pp. 72-85, 2006. 
[3] A.-M. Wazwaz, "Abundant solitons solutions for several forms of the fifth-order KdV equation by using the tanh method," Applied Mathematics and Computation, vol. 182, no. 1, pp. 283300, 2006.

[4] L. Huibin and W. Kelin, "Exact solutions for two nonlinear equations. I," Journal of Physics A: Mathematical and General, vol. 23 , no. 17 , pp. 3923-3928, 1990.

[5] W.-P. Hong and Y.-D. Jung, "Auto-Backlund transformation and solitary-wave solutions to nonintegrable generalized fifth-order nonlinear evolution equations," Zeitschrift für Naturforschung A, vol. 54, no. 8-9, pp. 549-553, 1999.

[6] A. Karasu-Kalkanli, A. Karasu, A. Sakovich, S. Sakovich, and R. Turhan, "A new integrable generalization of the Korteweg-de Vries equation," Journal of Mathematical Physics, vol. 49, no. 7, pp. 2634-2639, 2008.

[7] J. B. Li and Y. Zhang, "Homoclinic manifolds, center manifolds and exact solutions of four-dimensional traveling wave systems for two classes of nonlinear wave equations," International Journal of Bifurcation and Chaos, vol. 21, no. 2, pp. 527-543, 2011.

[8] L. J. Zhang and C. M. Khalique, "Exact solitary wave and periodic wave solutions of the Kaup-Kuperschmidt equation," Journal of Applied Analysis and Computation, vol. 5, no. 3, pp. 485-495, 2015.

[9] A. Parker, "On soliton solutions of the Kaup-Kupershmidt equation. I. Direct bilinearisation and solitary wave," Physica D: Nonlinear Phenomena, vol. 137, no. 1-2, pp. 25-33, 2000.

[10] A. Parker, "On soliton solutions of the Kaup-Kupershmidt equation. II. 'Anomalous' N-soliton solutions,' Physica D: Nonlinear Phenomena, vol. 137, no. 1-2, pp. 34-48, 2000.

[11] K. Sawada and T. Kotera, "A method for finding $N$-soliton solutions for the K.d.V. equation and K.d.V.-like equation," Progress of Theoretical Physics, vol. 51, no. 5, pp. 1355-1367, 1974.

[12] P. J. Caudrey, R. K. Dodd, and J. D. Gibbon, "A new hierarchy of Korteweg-de Vries equation," Proceedings of the Royal Society of London A, vol. 351, no. 1666, pp. 407-422, 1976.

[13] M. Ito, "An extension of nonlinear evolution equations of the $\mathrm{K}-\mathrm{dV}$ (mK-dV) type to higher orders," Journal of the Physical Society of Japan, vol. 49, no. 2, pp. 771-778, 1980.

[14] J. B. Li, Singular Traveling Wave Equations: Bifurcations and Exact Solutions, Science Press, Beijing, China, 2013.

[15] J. B. Li and Y. Zhang, "The exact traveling wave solutions to two integrable KdV6 equations," Chinese Annals of Mathematics B, vol. 33, no. 2, pp. 179-190, 2012.

[16] C. M. Cosgrove, "Higher-order Painlevé equations in the polynomial class I. Bureau symbol P2," Studies in Applied Mathematics, vol. 104, no. 1, pp. 1-65, 2000.

[17] W. Malfliet and W. Hereman, "The tanh method I: exact solutions of nonlinear evolution and wave equations," Physica Scripta, vol. 54, no. 6, pp. 563-568, 1996.

[18] C. T. Yan, "A simple transformation for nonlinear waves," Physics Letters, Section A: General, Atomic and Solid State Physics, vol. 224, no. 1-2, pp. 77-84, 1996.

[19] W. X. Ma and B. Fuchssteiner, "Explicit and exact solutions to a Kolmogorov-Petrovskii-Piskunov equation," International Journal of Non-Linear Mechanics, vol. 31, no. 3, pp. 329-338, 1996.

[20] E. J. Parkes, B. R. Duffy, and P. C. Abbott, "The Jacobi elliptic-function method for finding periodic-wave solutions to nonlinear evolution equations," Physics Letters A, vol. 295, no. 5-6, pp. 280-286, 2002.
[21] J.-H. He and X.-H. Wu, "Exp-function method for nonlinear wave equations," Chaos, Solitons and Fractals, vol. 30, no. 3, pp. 700-708, 2006.

[22] M. L. Wang, Y. B. Zhou, and Z. B. Li, "Application of a homogeneous balance method to exact solutions of nonlinear equations in mathematical physics," Physics Letters A, vol. 216, no. $1-5$, pp. $67-75,1996$.

[23] M. Wang, X. Li, and J. Zhang, “The $\left(G^{\prime} / G\right)$-expansion method and travelling wave solutions of nonlinear evolution equations in mathematical physics," Physics Letters A, vol. 372, no. 4, pp. 417-423, 2008.

[24] H. Q. Zhang, "New exact travelling wave solutions of nonlinear evolution equation using a sub-equation," Chaos, Solitons \& Fractals, vol. 39, no. 2, pp. 873-881, 2009.

[25] W.-X. Ma and J.-H. Lee, "A transformed rational function method and exact solutions to the $3+1$ dimensional JimboMiwa equation," Chaos, Solitons \& Fractals, vol. 42, no. 3, pp. 1356-1363, 2009.

[26] L. J. Zhang and X. W. Huo, "On the Exp-function method for constructing travelling wave solutions of nonlinear equations in nonlinear and modern mathematical physics," in Proceedings of the 1st International Workshop, vol. 1212, pp. 280-285, Beijing, China, 2010.

[27] N. A. Kudryashov, "Seven common errors in finding exact solutions of nonlinear differential equations," Communications in Nonlinear Science and Numerical Simulation, vol. 14, no. 9-10, pp. 3507-3529, 2009.

[28] X.-Y. Wen, Y.-T. Gao, and L. Wang, "Darboux transformation and explicit solutions for the integrable sixth-order KdV equation for nonlinear waves," Applied Mathematics and Computation, vol. 218, no. 1, pp. 55-60, 2011.

[29] L. J. Zhang, L.-Q. Chen, and X. W. Huo, "The effects of horizontal singular straight line in a generalized nonlinear KleinGordon model equation," Nonlinear Dynamics, vol. 72, no. 4, pp. 789-801, 2013.

[30] J. W. Shen, W. Xu, and W. Li, "Bifurcations of travelling wave solutions in a new integrable equation with peakon and compactons," Chaos, Solitons and Fractals, vol. 27, no. 2, pp. 413425, 2006.

[31] Y. Wang and Q. Bi, "Different wave solutions associated with singular lines on phase plane," Nonlinear Dynamics, vol. 69, no. 4, pp. 1705-1731, 2012.

[32] W. G. Rui, "Different kinds of exact solutions with two-loop character of the two-component short pulse equations of the first kind," Communications in Nonlinear Science and Numerical Simulation, vol. 18, no. 10, pp. 2667-2678, 2013.

[33] S. N. Chow and J. K. Hale, Method of Bifurcation Theory, Springer, New York, NY, USA, 1981.

[34] J. Guckenheimer and P. Holmes, Nonlinear Oscillations, Dynamical Systems, and Bifurcations of Vector Fields, Springer, New York, NY, USA, 1983. 


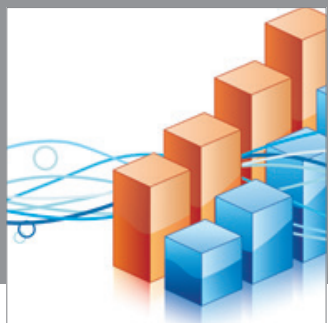

Advances in

Operations Research

mansans

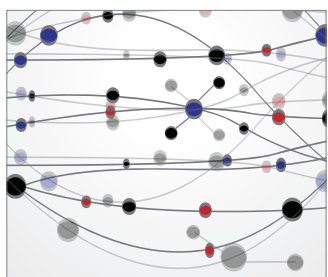

The Scientific World Journal
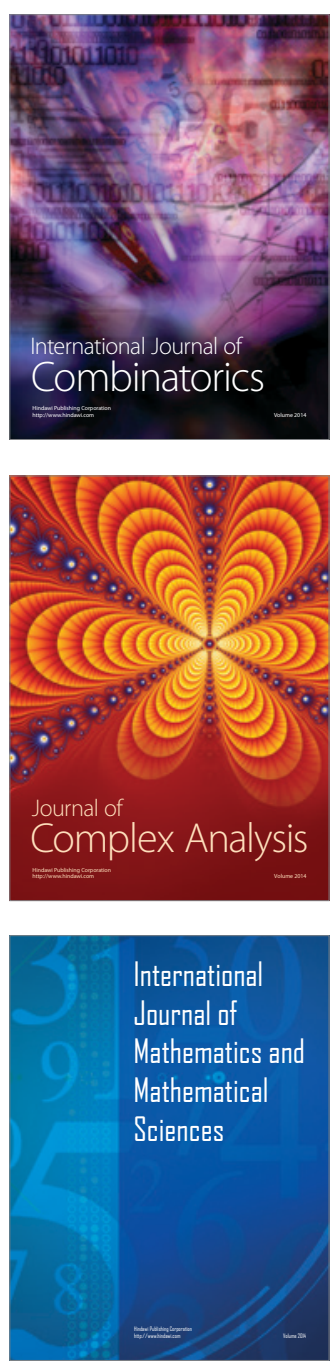
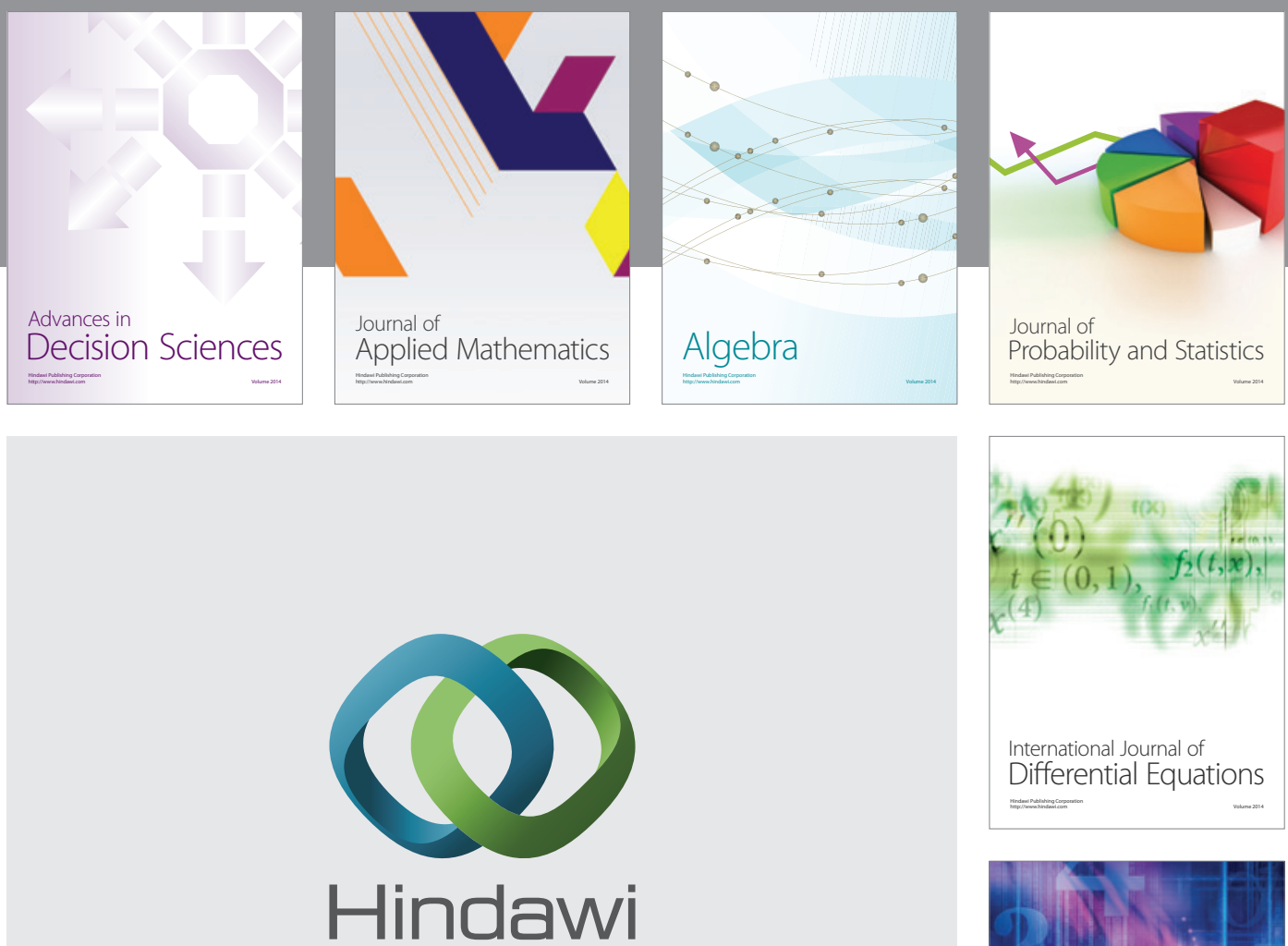

Submit your manuscripts at http://www.hindawi.com
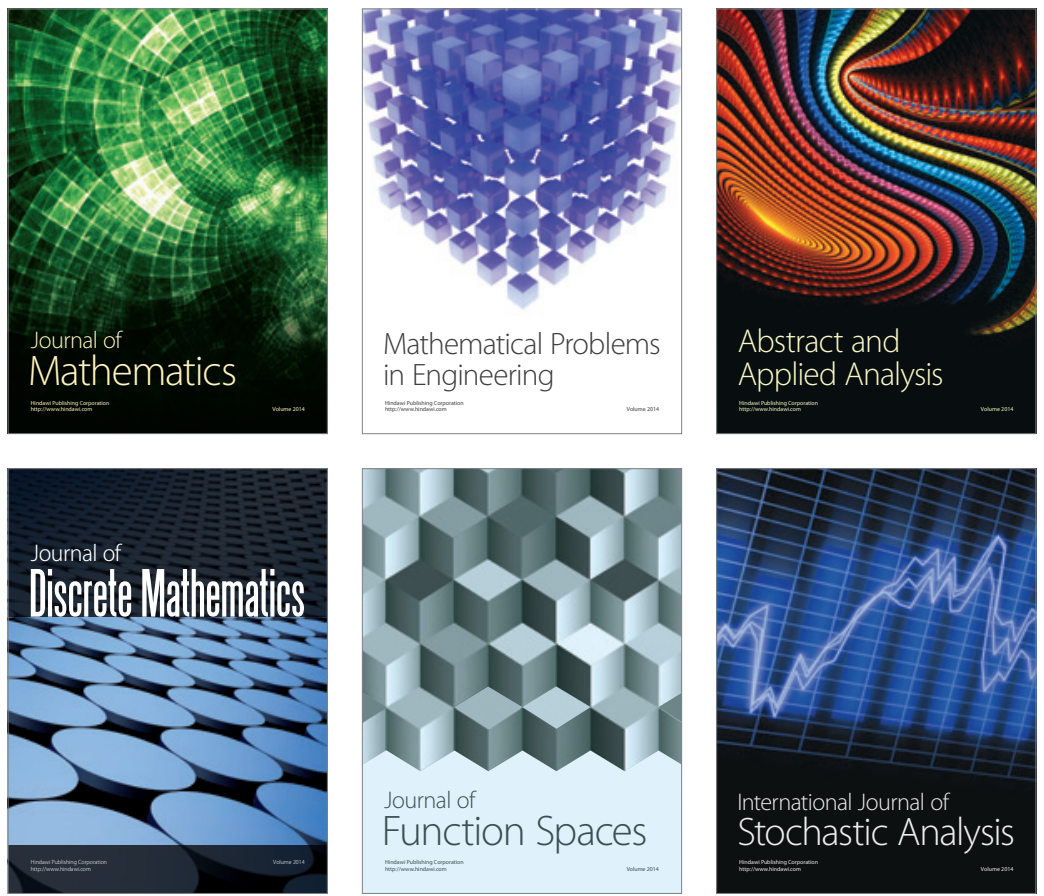

Journal of

Function Spaces

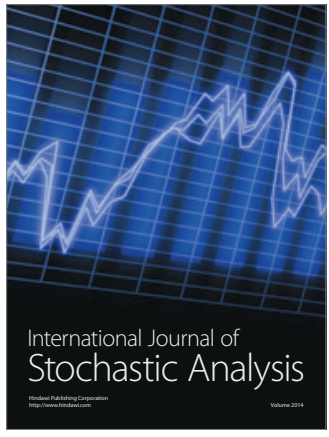

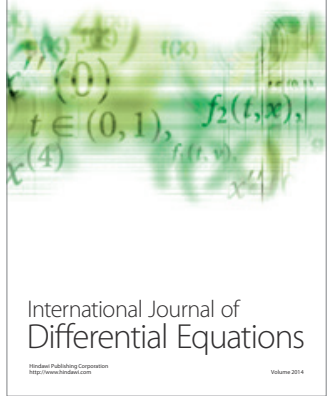
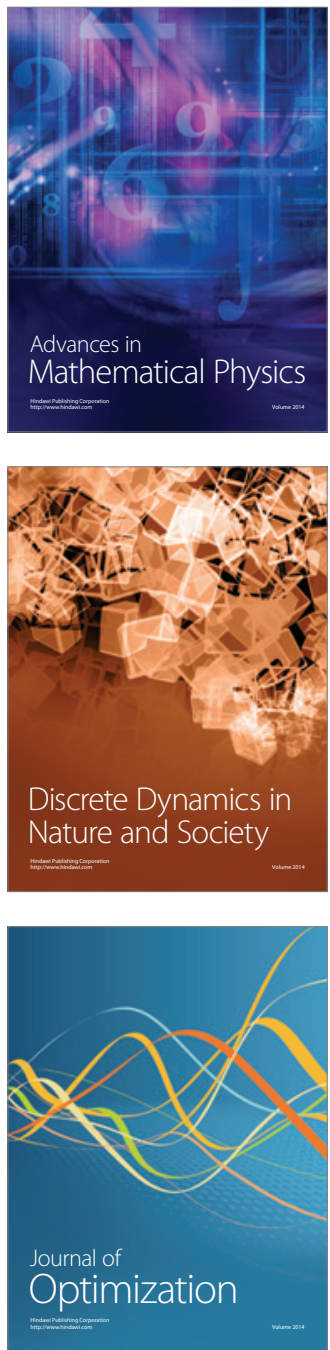